\title{
ON THE BRUNK-CHUNG TYPE STRONG LAW OF LARGE NUMBERS FOR SEQUENCES OF BLOCKWISE $m$-DEPENDENT RANDOM VARIABLES
}

\author{
LE VAN THANH ${ }^{1}$
}

\begin{abstract}
For a sequence of blockwise $m$-dependent random variables $\left\{X_{n}, n \geq 1\right\}$, conditions are provided under which $\lim _{n \rightarrow \infty}\left(\sum_{i=1}^{n} X_{i}\right) / b_{n}=0$ almost surely where $\left\{b_{n}, n \geq 1\right\}$ is a sequence of positive constants. The results are new even when $b_{n} \equiv n^{r}, r>0$. As special case, the BrunkChung strong law of large numbers is obtained for sequences of independent random variables. The current work also extends results of Móricz [Proc. Amer. Math. Soc. 101 (1987) 709-715], and Gaposhkin [Teor. Veroyatnost. i Primenen. 39 (1994) 804-812]. The sharpness of the results is illustrated by examples.
\end{abstract}

Mathematics Subject Classification. 60F15.

Received September 5, 2005.

\section{INTRODUCTION}

The Brunk-Chung strong law of large numbers (SLLN) (see, e.g., Chow and Teicher [2], p. 363) asserts for a sequence of independent random variables $\left\{X_{n}, n \geq 1\right\}$ with $E X_{n}=0, n \geq 1$ that the condition

$$
\sum_{n=1}^{\infty} \frac{E\left|X_{n}\right|^{2 p}}{n^{p+1}}<\infty(p \geq 1)
$$

is sufficient for the SLLN

$$
\lim _{n \rightarrow \infty} \frac{\sum_{i=1}^{n} X_{i}}{n}=0 \text { almost surely (a.s.). }
$$

Móricz [5] introduced the concept of blockwise independence for a sequence of random variables and extended a classical SLLN of Kolmogorov (see, e.g., Chow and Teicher [2], p. 124) to the blockwise $m$-dependent case. Gaposhkin [3] also studied the SLLN problem for sequences of blockwise independent random variables.

In the current work, we provide a Brunk-Chung type SLLN for sequences of blockwise $m$-dependent random variables $\left\{X_{n}, n \geq 1\right\}$. Conditions are provided for

$$
\lim _{n \rightarrow \infty} \frac{\sum_{i=1}^{n} X_{i}}{b_{n}}=0 \text { a.s. }
$$

Keywords and phrases. Strong law of large numbers, almost sure convergence, blockwise $m$-dependent random variables.

1 Department of Mathematics, Vinh University, Vinh, Nghe An 42118, Vietnam. 1vthanhvinh@yahoo.com 
to hold where $\left\{b_{n}, n \geq 1\right\}$ is a sequence of positive constants with $b_{n} \uparrow \infty$. The current work also extends results of Móricz [5], and Gaposhkin [3]. Our proofs are substantially simpler than those of the earlier counterparts thanks to a recent and elementary result of Chobanyan, Levental, and Mandrekar [1].

The plan of the paper is as follows. Technical definitions, notation, and the lemmas and other results used in the proofs of the main results or their corollaries are consolidated into Section 2. In Section 3, the main results, some corollaries and illustrative examples are presented.

\section{PRELIMINARIES}

Some definitions and preliminary results will be presented in prior to establishing the main result.

Let $m$ be a nonnegative integer. A finite collection of random variables $\left\{X_{1}, \ldots, X_{n}\right\}$ is said to be $m$-dependent if either $n \leq m+1$ or $n>m+1$ and the random variables $\left\{X_{1}, \ldots, X_{i}\right\}$ are independent of the random variables $\left\{X_{j}, \ldots, X_{n}\right\}$ whenever $j-i>m$. A sequence of random variables $\left\{X_{n}, n \geq 1\right\}$ is said to be $m$-dependent if for each $n \geq 1$, the random variables $\left\{X_{1}, \ldots, X_{n}\right\}$ are $m$-dependent.

Let $\left\{\beta_{k}, k \geq 1\right\}$ be a strictly increasing sequence of positive integers with $\beta_{1}=1$ and set $B_{k}=\left[\beta_{k}, \beta_{k+1}\right)$. A sequence of random variables $\left\{X_{n}, n \geq 1\right\}$ is said to be blockwise $m$-dependent with respect to the blocks $\left\{B_{k}, k \geq 1\right\}$ if for each $k \geq 1$, the random variables $\left\{X_{i}, i \in B_{k}\right\}$ are $m$-dependent.

The following notation will be used throughout this paper. For $x \geq 0$, let $[x]$ denote the greatest integer less than or equal to $x$. We use $\log$ to denote the logarithm to the base 2 . The symbol $C$ denotes a generic constant $(0<C<\infty)$ which is not necessarily the same one in each appearance.

For $\left\{\beta_{k}, k \geq 1\right\}$ and $\left\{B_{k}, k \geq 1\right\}$ as above, we introduce the following notation:

$$
\begin{aligned}
B^{(l)} & =\left\{k: 2^{l} \leq k<2^{l+1}\right\}, l \geq 0, \\
B_{k}^{(l)} & =B_{k} \cap B^{(l)}, k \geq 1, l \geq 0, \\
I_{l} & =\left\{k \geq 1: B_{k}^{(l)} \neq \emptyset\right\}, l \geq 0, \\
r_{k}^{(l)} & =\min \left\{r: r \in B_{k}^{(l)}\right\}, k \in I_{l}, l \geq 0, \\
c_{l} & =\operatorname{card} I_{l}, l \geq 0, \\
d_{l} & =\max _{k \in I_{l}} \operatorname{card} B_{k}^{(l)}, l \geq 0, \\
\varphi(n) & =\sum_{l=0}^{\infty} c_{l} I_{B^{(l)}}(n), n \geq 1, \\
\phi(n) & =\sum_{l=0}^{\infty} d_{l} I_{B^{(l)}}(n), n \geq 1, \\
\psi(n) & =\max _{k \leq n} \varphi(k), n \geq 1
\end{aligned}
$$

where $I_{B^{(l)}}$ denotes the indicator function of the set $B^{(l)}, l \geq 0$.

It is easy to verify that the following relations prevail.

(i) If $\beta_{k}=2^{k-1}, k \geq 1$, then

$$
s_{l}=1, l \geq 0, \varphi(n)=1, n \geq 1, \text { and } n / 2 \leq \phi(n) \leq n, n \geq 1 .
$$

(ii) If $\beta_{k}=\left[q^{k-1}\right]$ for all large $k$ where $q>1$, then

$$
s_{l}=\mathcal{O}(1), \varphi(n)=\mathcal{O}(1), \text { and } \phi(n)=\mathcal{O}(n) .
$$


(iii) If $\beta_{k}=\left[2^{(k-1)^{\alpha}}\right]$ for all large $k$ where $0<\alpha<1$, then

$$
s_{l}=\mathcal{O}\left(l^{(1-\alpha) / \alpha}\right), \varphi(n)=\mathcal{O}\left((\log n)^{(1-\alpha) / \alpha}\right), \text { and } \phi(n)=\mathcal{O}\left(n(\log n)^{\alpha-1}\right) .
$$

(iv) If $\beta_{k}=\left[k^{\alpha}\right], k \geq 1$ where $\alpha>1$, then

$$
s_{l}=\mathcal{O}\left(2^{l / \alpha}\right) \text { and } \varphi(n)=\mathcal{O}\left(n^{1 / \alpha}\right) \text {, and } \phi(n)=\mathcal{O}\left(n^{(\alpha-1) / \alpha}\right) .
$$

(v) If $\beta_{k}=k, k \geq 1$, then

$$
s_{l}=2^{l}, l \geq 0, n / 2 \leq \varphi(n) \leq n, n \geq 1, \text { and } \phi(n)=1, n \geq 1 .
$$

(vi) For any sequence $\left\{\beta_{k}, k \geq 1\right\}$

$$
\varphi(n) \leq n, n \geq 1, \text { and } \phi(n) \leq n, n \geq 1 .
$$

Lemma 2.1. Let $\left\{X_{n}, n \geq 1\right\}$ be a sequence of independent random variables with $E X_{n}=0, n \geq 1$ and let $p>1$. Then

$$
E\left(\left(\max _{1 \leq j \leq n}\left|\sum_{i=1}^{j} X_{i}\right|\right)^{p}\right) \leq C E\left(\sum_{i=1}^{n} X_{i}^{2}\right)^{p / 2}
$$

where the constant $C$ does not depend on $n$.

Proof. By Doob's submartingale maximal inequality (see, e.g., Chow and Teicher [2], p. 255)) and the Marcinkiewicz-Zygmund inequality (see, e.g., Chow and Teicher [2], p. 386)), for $n \geq 1$

$$
\begin{aligned}
E\left(\left(\max _{1 \leq j \leq n}\left|\sum_{i=1}^{j} X_{i}\right|\right)^{p}\right) & \leq\left(\frac{p}{p-1}\right)^{p} E\left|\sum_{i=1}^{n} X_{i}\right|^{p} \\
& \leq C E\left(\sum_{i=1}^{n} X_{i}^{2}\right)^{p / 2}
\end{aligned}
$$

establishing (2.7).

Lemma 2.2. Let $\left\{X_{i}, 1 \leq i \leq n\right\}$ be a collection of $m$-dependent random variables with $E X_{i}=0,1 \leq i \leq n$ and let $p \geq 1$. Then there exists a constant $C$ depending only on $m$ and $p$ such that

$$
\begin{aligned}
& E\left(\left(\max _{1 \leq j \leq n}\left|\sum_{i=1}^{j} X_{i}\right|\right)^{p}\right) \leq C \sum_{i=1}^{n} E\left|X_{i}\right|^{p}, \quad 1 \leq p \leq 2 . \\
& E\left(\left(\max _{1 \leq j \leq n}\left|\sum_{i=1}^{j} X_{i}\right|\right)^{p}\right) \leq C n^{p / 2-1} \sum_{i=1}^{n} E\left|X_{i}\right|^{p}, \quad p \geq 2 .
\end{aligned}
$$

Proof. If $n \leq m+1$, then Lemma 2.2 is trivial. So let $n>m+1$. 
In the case $p=1$, note that for all $n \geq 1$

$$
\begin{aligned}
E\left(\max _{1 \leq j \leq n}\left|\sum_{i=1}^{j} X_{i}\right|\right) & \leq E\left(\max _{1 \leq j \leq n} \sum_{i=1}^{j}\left|X_{i}\right|\right) \\
& =E\left(\sum_{i=1}^{n}\left|X_{i}\right|\right) \\
& =\sum_{i=1}^{n} E\left|X_{i}\right|
\end{aligned}
$$

establishing (2.8).

In the case $p>1$, for $n \geq m+1$

$$
\begin{aligned}
E\left(\left(\max _{1 \leq j \leq n}\left|\sum_{i=1}^{j} X_{i}\right|\right)^{p}\right) & \leq E\left(\left(\sum_{j=1}^{m+1} \max _{0 \leq k(m+1) \leq n-j}\left|\sum_{i=0}^{k} X_{i(m+1)+j}\right|\right)^{p}\right) \\
& \leq(m+1)^{p-1} \sum_{j=1}^{m+1} E\left(\left(\max _{0 \leq k(m+1) \leq n-j}\left|\sum_{i=0}^{k} X_{i(m+1)+j}\right|\right)^{p}\right) \\
& \leq C \sum_{j=1}^{m+1} E\left(\left(\sum_{0 \leq i(m+1) \leq n-j} X_{i(m+1)+j}^{2}\right)^{p / 2}\right) \text { (by Lem. 2.1). }
\end{aligned}
$$

If $1<p \leq 2$, then

$$
\begin{aligned}
\sum_{j=1}^{m+1} E\left(\left(\sum_{0 \leq i(m+1) \leq n-j} X_{i(m+1)+j}^{2}\right)^{p / 2}\right) & \leq \sum_{j=1}^{m+1} E\left(\sum_{0 \leq i(m+1) \leq n-j}\left|X_{i(m+1)+j}\right|^{p}\right) \\
& =\sum_{i=1}^{n} E\left|X_{i}\right|^{p} .
\end{aligned}
$$

The conclusion (2.8) follows immediately from (2.10) and (2.11).

If $p \geq 2$, then

$$
\begin{aligned}
\sum_{j=1}^{m+1} E\left(\left(\sum_{0 \leq i(m+1) \leq n-j} X_{i(m+1)+j}^{2}\right)^{p / 2}\right) & \leq n^{p / 2-1} \sum_{j=1}^{m+1} E\left(\sum_{0 \leq i(m+1) \leq n-j}\left|X_{i(m+1)+j}\right|^{p}\right) \\
& (\text { since }(n-j) /(m+1) \leq n, j=1, \ldots, m+1) \\
& =n^{p / 2-1} \sum_{i=1}^{n} E\left|X_{i}\right|^{p} .
\end{aligned}
$$

The conclusion (2.9) follows immediately from (2.10) and (2.12).

Lemma 2.3 (Chobanyan, Levental, and Mandrekar [1]). Let $\left\{X_{n}, n \geq 1\right\}$ be a sequence of random variables, let $\left\{b_{n}, n \geq 1\right\}$ be a nondecreasing sequence of positive constants, and let $\left\{k_{n}, n \geq 0\right\}$ be a sequence of positive integers such that

$$
\inf _{n \geq 0} \frac{b_{k_{n+1}}}{b_{k_{n}}}>1 \text { and } \sup _{n \geq 0} \frac{b_{k_{n+1}}}{b_{k_{n}}}<\infty
$$


Then

$$
\lim _{n \rightarrow \infty} \frac{\sum_{i=1}^{n} X_{i}}{b_{n}}=0 \text { a.s. }
$$

if and only if

$$
\lim _{n \rightarrow \infty} \frac{\max _{k_{n} \leq k<k_{n+1}}\left|\sum_{i=k_{n}}^{k} X_{i}\right|}{b_{k_{n+1}}-b_{k_{n}}}=0 \text { a.s. }
$$

\section{Remark 2.4.}

(i) Note that the first half of (2.13) ensures that $\left\{k_{n}, n \geq 0\right\}$ is strictly increasing and $\lim _{n \rightarrow \infty} b_{n}=\infty$.

(ii) It follows that if (2.15) holds for some sequence of positive integers $\left\{k_{n}, n \geq 0\right\}$ satisfying (2.13), then (2.15) holds for every sequence of positive integers $\left\{k_{n}, n \geq 0\right\}$ satisfying (2.13). Thus, in order to prove the SLLN (2.14), nothing is lost in working with a convenient sequence such as $k_{n}=2^{n}, n \geq 0$. This remark was made by Chobanyan, Levental, and Mandrekar [1].

\section{Results}

With the preliminaries accounted for, the main results may now be established. When $p=1, m=0$, and $b_{n} \equiv n$, Theorem 3.1 was obtained by Gaposhkin [3] using a substantially more complicated argument. Gaposhkin's [3] result is an extension of an earlier result of Móricz [5] which was apparently the first SLLN for a sequence of blockwise $m$-dependent random variables.

Theorem 3.1. Let $\left\{X_{n}, n \geq 1\right\}$ be a sequence of random variables with $E X_{n}=0, n \geq 1$, let $p \geq 1$ and let $\left\{b_{n}, n \geq 1\right\}$ be a nondecreasing sequence of positive constants such that

$$
\inf _{n \geq 0} \frac{b_{2^{n+1}}}{b_{2^{n}}}>1 \text { and } \sup _{n \geq 0} \frac{b_{2^{n+1}}}{b_{2^{n}}}<\infty .
$$

If $\left\{X_{n}, n \geq 1\right\}$ is blockwise $m$-dependent with respect to the blocks $\left\{B_{k}, k \geq 1\right\}$ and if

$$
\sum_{n=1}^{\infty} \frac{E\left|X_{n}\right|^{2 p}}{b_{n}^{2 p}}(\varphi(n))^{2 p-1}(\phi(n))^{p-1}<\infty,
$$

then the SLLN

$$
\lim _{n \rightarrow \infty} \frac{1}{b_{n}} \sum_{i=1}^{n} X_{i}=0 \text { a.s. }
$$

obtains.

Proof. Set

and

$$
T_{k}^{(l)}=\max _{j \in B_{k}^{(l)}}\left|\sum_{i=r_{k}^{(m)}}^{j} X_{i}\right|, k \in I_{l}, l \geq 0
$$

$$
T_{l}=\frac{1}{b_{2^{l+1}}} \sum_{k \in I_{l}} T_{k}^{(l)}, l \geq 0 .
$$


Note that for $l \geq 0$

$$
\begin{aligned}
E\left(T_{l}\right)^{2 p} & \leq \frac{1}{b_{2^{2+1}}^{2 p}} c_{l}^{2 p-1} \sum_{k \in I_{l}} E\left(T_{k}^{(l)}\right)^{2 p} \\
& \leq \frac{1}{b_{2^{l+1}}^{2 p}} c_{l}^{2 p-1} C \sum_{k \in I_{l}} \sum_{i \in B_{k}^{(l)}}\left(\operatorname{card} B_{k}^{(l)}\right)^{p-1} E\left|X_{i}\right|^{2 p} \quad \text { by Lem. 2.2) } \\
& \leq \frac{1}{b_{2^{2+1}}^{2 p}} c_{l}^{2 p-1} d_{l}^{p-1} \sum_{i=2^{l}}^{2^{l+1}-1} E\left|X_{i}\right|^{2 p} \\
& \leq C \sum_{i=2^{l}}^{2^{l+1}-1} \frac{E\left|X_{i}\right|^{2 p}}{b_{i}^{2 p}}(\varphi(i))^{2 p-1}(\phi(i))^{p-1}
\end{aligned}
$$

It follows from (3.2) that $\sum_{l=0}^{\infty} E T_{l}^{2 p}<\infty$ and so by the Markov inequality and the Borel-Cantelli lemma

$$
\lim _{l \rightarrow \infty} T_{l}=0 \text { a.s. }
$$

Now it follows from the first half of (3.1) that

$$
\frac{\max _{2^{l} \leq k<2^{l+1}}\left|\sum_{i=2^{l}}^{k} X_{i}\right|}{b_{2^{l+1}}-b_{2^{l}}} \leq \frac{C \max _{2^{l} \leq k<2^{l+1}}\left|\sum_{i=2^{l}}^{k} X_{i}\right|}{b_{2^{l+1}}} \leq C T_{l} \rightarrow 0 \text { a.s. }
$$

The conclusion (3.3) follow immediately from Lemma 2.3.

\section{Remark 3.2.}

(i) The slower $b_{n} \uparrow \infty$, the stronger is the assumption (3.2) but so is the conclusion (3.3).

(ii) When (3.1) holds where $0<b_{n} \uparrow, n=\mathcal{O}\left(b_{n}\right)$ and

$$
c_{l}=o\left(a^{l}\right) \text { for all } a>1
$$

then a sufficient condition for (3.2) to hold is that

$$
\sum_{n=1}^{\infty} \frac{E\left|X_{n}\right|^{2 p}}{b_{n}^{q+1}}<\infty \text { for some } 0<q<p
$$

To see this, it follows from the first half of (3.1) that $b_{2^{n+1}} / b_{2^{n}} \geq 1+\delta$ for some $\delta>0$ and all $n \geq 0$. Thus for all $l \geq 1$,

$$
b_{2^{l}}=b_{1} \prod_{j=0}^{l-1} \frac{b_{2^{j+1}}}{b_{2^{j}}} \geq b_{1}(1+\delta)^{l}
$$


Hence

$$
\begin{aligned}
\sum_{n=1}^{\infty} \frac{E\left|X_{n}\right|^{2 p}}{b_{n}^{2 p}(\varphi(n))^{2 p-1}(\phi(n))^{p-1}} & \leq \sum_{n=1}^{\infty} \frac{E\left|X_{n}\right|^{2 p}}{b_{n}^{p+1}}(\varphi(n))^{2 p-1}\left(\frac{n}{b_{n}}\right)^{p-1} \quad(\text { by }(2.6)) \\
& \leq C \sum_{n=1}^{\infty} \frac{E\left|X_{n}\right|^{2 p}}{b_{n}^{p+1}}(\varphi(n))^{2 p-1}\left(\text { since } n=\mathcal{O}\left(b_{n}\right)\right) \\
& =C \sum_{l=0}^{\infty} \sum_{i=2^{l}}^{2^{l+1}-1} \frac{E\left|X_{i}\right|^{2 p}(\varphi(i))^{2 p-1}}{b_{i}^{q+1} b_{i}^{p-q}} \\
& \leq C \sum_{l=0}^{\infty} \sum_{i=2^{l}}^{2^{l+1}-1} \frac{E\left|X_{i}\right|^{2 p} c_{l}^{2 p-1}}{b_{i}^{q+1} b_{2^{l}}^{p-q}} \\
& \leq C \sum_{l=0}^{\infty} \sum_{i=2^{l}}^{2^{l+1}-1} \frac{E\left|X_{i}\right|^{2 p} c_{l}^{2 p-1}}{b_{i}^{q+1} b_{1}^{p-q}(1+\delta)^{(p-q) l}}(\text { by }(3.6)) \\
& \leq C \sum_{l=0}^{\infty} \sum_{i=2^{l}}^{2^{l+1}-1} \frac{E\left|X_{i}\right|^{2 p}}{b_{i}^{q+1}} \\
& \left(\mathrm{by}(3.4) \text { with } a=(1+\delta)^{(p-q) /(2 p-1)}\right) \\
& =C \sum_{n=1}^{\infty} \frac{E\left|X_{n}\right|^{2 p}}{b_{n}^{q+1}}<\infty(\mathrm{by}(3.5))
\end{aligned}
$$

thereby establishing (3.2).

We next present alternative version of Theorem 3.1 .

Theorem 3.3. Let $\left\{X_{n}, n \geq 1\right\}$ be a sequence of random variables with $E X_{n}=0, n \geq 1$, let $p \geq 1$ and let $\left\{b_{n}, n \geq 1\right\}$ be a nondecreasing sequence of positive constants satisfying (3.1). If $\left\{X_{n}, n \geq 1\right\}$ is blockwise $m$-dependent with respect to the blocks $\left\{B_{k}, k \geq 1\right\}$ and if

$$
\sum_{n=1}^{\infty} \frac{E\left|X_{n}\right|^{2 p}}{b_{n}^{2 p}}(\phi(n))^{p-1}<\infty
$$

then the SLLN

$$
\lim _{n \rightarrow \infty} \frac{\sum_{i=1}^{n} X_{i}}{b_{n}(\psi(n))^{(2 p-1) /(2 p)}}=0 \text { a.s. }
$$

obtains.

Proof. Define $T_{k}^{(l)}, k \in I_{l}, l \geq 0$ as in the proof of Theorem 3.1 and set

$$
\tau_{l}=\frac{1}{\left(b_{2^{l+1}}-b_{2^{l}}\right)\left(\psi\left(2^{l}\right)\right)^{(2 p-1) /(2 p)}} \sum_{k \in I_{l}} T_{k}^{(l)}, l \geq 0
$$


Note that for $l \geq 0$

$$
\begin{aligned}
E \tau_{l}^{2 p} & \left.\leq \frac{C}{b_{2^{l+1}}^{2 p}\left(\psi\left(2^{l}\right)\right)^{2 p-1}} c_{l}^{2 p-1} \sum_{k \in I_{l}} E\left(T_{k}^{(l)}\right)^{2 p} \text { (by the first half of }(3.1)\right) \\
& \leq \frac{C}{b_{2^{2+1}}^{2 p}} \sum_{k \in I_{l}} E\left(T_{k}^{(l)}\right)^{2 p} \\
& \leq \frac{C}{b_{2^{l+1}}^{2 p}} \sum_{k \in I_{l}} \sum_{i \in B_{k}^{(l)}}\left(\operatorname{card} B_{k}^{(l)}\right)^{p-1} E\left|X_{i}\right|^{2 p} \text { (by Lem. 2.2) } \\
& \leq \frac{C}{b_{2^{2+1}}^{2 p}} d_{l}^{p-1} \sum_{i=2^{l}}^{2^{l+1}-1} E\left|X_{i}\right|^{2 p} \\
& \leq C \sum_{i=2^{l}} \frac{E\left|X_{i}\right|^{2 p}}{b_{i}^{2 p}}(\phi(i))^{p-1}
\end{aligned}
$$

It follows from (3.7) that $\sum_{l=0}^{\infty} E \tau_{l}^{2 p}<\infty$ and so by the Markov inequality and the Borel-Cantelli lemma

$$
\lim _{l \rightarrow \infty} \tau_{l}=0 \text { a.s. }
$$

Note that for $n \geq 1$, letting $M \geq 0$ be such that $2^{M} \leq n<2^{M+1}$,

$$
\begin{aligned}
\frac{\left|\sum_{i=1}^{n} X_{i}\right|}{b_{n}(\psi(n))^{(2 p-1) /(2 p)}} & \leq \frac{\sum_{l=0}^{M} \sum_{k \in I_{l}} T_{k}^{(l)}}{b_{2^{M}}\left(\psi\left(2^{M}\right)\right)^{(2 p-1) /(2 p)}} \\
& \leq \sum_{l=0}^{M} \frac{b_{2^{l+1}}-b_{2^{l}}}{b_{2^{M}}} \tau_{l}
\end{aligned}
$$

The conclusion (3.8) follow immediately from the second half of (3.1), (3.9), (3.10) and the Toeplitz lemma (see, e.g., Loève [4], p. 250).

Some particular cases may now be presented as corollaries. When $p=1, m=0$, and $b_{n} \equiv n$, Corollaries 3.4, 3.5, and 3.6 reduce to results of Gaposhkin [3]. This special case of Corollary 3.4 is also the SLLN for a sequence of blockwise $m$-dependent random variables obtained by Móricz [5].

Corollary 3.4. Let $\left\{X_{n}, n \geq 1\right\}$ be a sequence of random variables with $E X_{n}=0, n \geq 1$ and let $\left\{b_{n}, n \geq 1\right\}$ be a nondecreasing sequence of positive constants such that (3.1) holds. If $\left\{X_{n}, n \geq 1\right\}$ is blockwise m-dependent with respect to the blocks $\left\{\left[2^{k-1}, 2^{k}\right), k \geq 1\right\}$ (or, more generally, with respect to the blocks $\left\{\left[\beta_{k}, \beta_{k+1}\right), k \geq 1\right\}$ where $\beta_{k}=\left[q^{k-1}\right]$ for all large $k$ and $q>1$ ) and if

$$
\sum_{n=1}^{\infty} \frac{n^{p-1} E\left|X_{n}\right|^{2 p}}{b_{n}^{2 p}}<\infty
$$

then the $S L L N$ (3.3) obtains.

Proof. Recalling (2.1) and (2.2), the assumption (3.11) ensures that (3.2) holds. The conclusion follows directly from Theorem 3.1.

Corollary 3.5. Let $\left\{X_{n}, n \geq 1\right\}$ be a sequence of random variables with $E X_{n}=0, n \geq 1$ and let $\left\{b_{n}, n \geq 1\right\}$ be a nondecreasing sequence of positive constants such that (3.1) holds. If $\left\{X_{n}, n \geq 1\right\}$ is blockwise m-dependent 
with respect to the blocks $\left\{\left[\beta_{k}, \beta_{k+1}\right), k \geq 1\right\}$ where $\beta_{k}=\left[2^{(k-1)^{\alpha}}\right]$ for all large $k$ where $0<\alpha<1$ and if

$$
\sum_{n=1}^{\infty} \frac{n^{p-1} E\left|X_{n}\right|^{2 p}}{b_{n}^{2 p}}(\log n)^{(1-\alpha)(2 p+\alpha-\alpha p-1) / \alpha}<\infty
$$

then the SLLN (3.3) obtains.

Proof. Recalling (2.3), the assumption (3.12) ensures that (3.2) holds. The conclusion follows directly from Theorem 3.1.

Corollary 3.6. Let $\left\{X_{n}, n \geq 1\right\}$ be a sequence of random variables with $E X_{n}=0, n \geq 1$ and let $\left\{b_{n}, n \geq 1\right\}$ be a nondecreasing sequence of positive constants such that (3.1) holds. If $\left\{X_{n}, n \geq 1\right\}$ is blockwise m-dependent with respect to the blocks $\left\{\left[\beta_{k}, \beta_{k+1}\right), k \geq 1\right\}$ where $\beta_{k}=\left[k^{\alpha}\right]$ for all large $k$ where $\alpha>1$ and if

$$
\sum_{n=1}^{\infty} \frac{n^{(p \alpha+p-\alpha) / \alpha} E\left|X_{n}\right|^{2 p}}{b_{n}^{2 p}}<\infty,
$$

then the SLLN (3.3) obtains.

Proof. Recalling (2.4), the assumption (3.13) ensures that (3.2) holds. The conclusion follows directly from Theorem 3.1.

The following corollary extends the Brunk-Chung strong law of large numbers to the blockwise $m$-dependent case.

Corollary 3.7. Let $\left\{X_{n}, n \geq 1\right\}$ be a sequence of random variables with $E X_{n}=0, n \geq 1$. If $\left\{X_{n}, n \geq 1\right\}$ is blockwise $m$-dependent with respect to the blocks $\left\{B_{k}, k \geq 1\right\}$ and if

$$
\sum_{n=1}^{\infty} \frac{E\left|X_{n}\right|^{2 p}}{n^{p+1}}(\varphi(n))^{2 p-1}<\infty
$$

then the $S L L N$

$$
\lim _{n \rightarrow \infty} \frac{\sum_{i=1}^{n} X_{i}}{n}=0 \text { a.s. }
$$

obtains.

Proof. Recalling (2.6), the assumption (3.14) ensures that (3.2) holds for $b_{n}=n, n \geq 1$. The conclusion (3.15) follows directly from Theorem 3.1.

We close by presenting two examples. These examples show that Theorem 3.1 can fail if the series in (3.2) diverges. More specifically, Examples 3.8 shows that we can not replace (3.2) by the weaker condition $\left(E\left|X_{n}\right|^{2 p}(\varphi(n))^{2 p-1}(\phi(n))^{p-1}\right) / b_{n}^{2 p}=o(1)$.

Example 3.8. Let $b_{n} \equiv n$ and let $X$ be a random variables with $P\{X=1\}=P\{X=-1\}=1 / 2$. Set $X_{n}=X, n \geq 1$. Then $\left\{X_{n}, n \geq 1\right\}$ is blockwise $m$-dependent with respect to the blocks $\{[k, k+1), k \geq 1\}$. Recalling (2.5),

$$
\begin{aligned}
\sum_{n=1}^{\infty} \frac{E\left|X_{n}\right|^{2 p}}{n^{2 p}}(\varphi(n))^{2 p-1}(\phi(n))^{p-1} & \geq C \sum_{n=1}^{\infty} \frac{1}{n^{2 p}} n^{2 p-1} \\
& =\infty
\end{aligned}
$$


and so (3.2) fails. Note that for all $n \geq 1$

$$
\frac{\sum_{i=1}^{n} X_{i}}{n}=\frac{n X}{n}=X \neq 0 \text { a.s. }
$$

Thus (3.3) fails. Again recalling (2.5),

$$
\begin{aligned}
\frac{E\left|X_{n}\right|^{2 p}}{n^{2 p}}(\varphi(n))^{2 p-1}(\phi(n))^{p-1} & =C \frac{1}{n^{2 p}} n^{2 p-1} \\
& =\frac{C}{n} \rightarrow 0 \text { as } n \rightarrow \infty .
\end{aligned}
$$

As Example 3.8, Example 3.9 also shows that Theorem 3.1 can fail if the series in (3.2) diverges.

Example 3.9. Let $p \geq 1$, and let $\left\{Y_{n}, n \geq 1\right\}$ be a sequence of 0 -dependent random variables such that $E Y_{n}=0, n \geq 1$ and

$$
P\left\{\left|Y_{n}\right|=(2 n-1)^{(p+1) /(2 p)}\right\}=\frac{1}{1+\log n}, P\left\{\left|Y_{n}\right|=0\right\}=1-\frac{1}{1+\log n}, n \geq 1 .
$$

Let $X_{n}=Y_{n-2^{l}+1}, 2^{l} \leq n<2^{l+1}, l \geq 0$ and let $b_{n}=n^{(p+1) /(2 p)}, n \geq 1$. Then $E X_{n}=0, n \geq 1$ and $\left\{X_{n}, n \geq 1\right\}$ is blockwise 0-dependent with respect to the blocks $\left\{\left[2^{k-1}, 2^{k}\right), k \geq 1\right\}$. Now $X_{2^{l+1}-1}=Y_{2^{l}}, l \geq 0$ and so $\left\{X_{2^{l+1}-1}, l \geq 0\right\}$ is a sequence of independent random variables with

$$
P\left\{\left|X_{2^{l+1}-1}\right|=\left(2^{l+1}-1\right)^{(p+1) /(2 p)}\right\}=\frac{1}{l+1}, P\left\{X_{2^{l+1}-1}=0\right\}=\frac{l}{l+1}, l \geq 0 .
$$

Then recalling (2.1),

$$
\begin{aligned}
\sum_{n=1}^{\infty} \frac{E\left|X_{n}\right|^{2 p}}{b_{n}^{2 p}}(\varphi(n))^{2 p-1}(\phi(n))^{p-1} & \geq C \sum_{n=1}^{\infty} \frac{E\left|X_{n}\right|^{2 p}}{n^{p+1}} n^{p-1} \\
& \geq C \sum_{l=0}^{\infty} \frac{E\left|X_{2^{l+1}-1}\right|^{2 p}}{\left(2^{l+1}-1\right)^{2}} \\
& =C \sum_{l=0}^{\infty} \frac{\left(2^{l+1}-1\right)^{p+1}}{(l+1)\left(2^{l+1}-1\right)^{2}}=\infty
\end{aligned}
$$

and so (3.2) fails. Also since

$$
\sum_{l=0}^{\infty} P\left\{\left|X_{2^{l+1}-1}\right|=\left(2^{l+1}-1\right)^{(p+1) /(2 p)}\right\}=\sum_{l=0}^{\infty} \frac{1}{l+1}=\infty
$$

by the Borel-Cantelli lemma

$$
P\left\{\left|X_{2^{l+1}-1}\right|=\left(2^{l+1}-1\right)^{(p+1) /(2 p)} \text { i.o. }(l)\right\}=1 .
$$

Thus,

$$
\begin{aligned}
1 & =\limsup _{l \rightarrow \infty} \frac{\left|X_{2^{l+1}-1}\right|}{\left(2^{l+1}-1\right)^{(p+1) /(2 p)}} \leq \limsup _{n \rightarrow \infty} \frac{\left|X_{n}\right|}{n^{(p+1) /(2 p)}} \\
& \leq \limsup _{n \rightarrow \infty} \frac{\left|\sum_{i=1}^{n} X_{i}\right|}{n^{(p+1) /(2 p)}}+\limsup _{n \rightarrow \infty} \frac{\left|\sum_{i=1}^{n-1} X_{i}\right|}{(n-1)^{(p+1) /(2 p)}} \text { a.s. }
\end{aligned}
$$


implying

Thus (3.3) fails.

$$
\limsup _{n \rightarrow \infty} \frac{\left|\sum_{i=1}^{n} X_{i}\right|}{b_{n}}=\limsup _{n \rightarrow \infty} \frac{\left|\sum_{i=1}^{n} X_{i}\right|}{n^{(p+1) /(2 p)}} \geq \frac{1}{2} \text { a.s. }
$$

Acknowledgements. The author is grateful to Professors Nguyen Duy Tien (Viet Nam National University, Ha Noi) and Andrew Rosalsky (University of Florida, USA) for some helpful and important remarks. The author also wishes to thank to Department of Mathematics at the Vinh University (Vietnam), especially to Professor Nguyen Van Quang for their attention and interest in my work.

\section{REFERENCES}

[1] S. Chobanyan, S. Levental and V. Mandrekar, Prokhorov blocks and strong law of large numbers under rearrangements. $J$. Theoret. Probab. 17 (2004) 647-672.

[2] Y.S. Chow and H. Teicher, Probability Theory: Independence, Interchangeability, Martingales. 3rd ed. Springer-Verlag, New York (1997).

[3] V.F. Gaposhkin, On the strong law of large numbers for blockwise independent and blockwise orthogonal random variables. Teor. Veroyatnost. i Primenen. 39 (1994) 804-812 (in Russian). English translation in Theory Probab. Appl. 39 (1994) 667-684 (1995).

[4] M. Loève, Probability Theory I. 4th ed. Springer-Verlag, New York (1977).

[5] F. Móricz, Strong limit theorems for blockwise $m$-dependent and blockwise quasiorthogonal sequences of random variables. Proc. Amer. Math. Soc. 101 (1987) 709-715. 